\title{
An infinitesimal trace formula for the Laplace operator on compact Riemann surfaces
}

François Golse and Pierre Lochak

\begin{abstract}
We derive an infinitesimal (or variational) version of the Selberg trace formula for compact Riemann surfaces, which gives information on the behaviour of the eigenvalues of the Laplace-Beltrami operator as the surface varies over the appropriate moduli space.
\end{abstract}

Mathematics Subject Classification (2000). 11F72, 32 G34 35P05.

Keywords. Riemann surfaces, Selberg trace formula, moduli spaces of curves.

\section{Introduction}

In this note we derive a variational form of the Selberg trace formula for compact Riemann surfaces, as the surface varies over the appropriate moduli space. We use the real analytic theory of moduli spaces, taking advantage in particular of Wolpert's variational formula $([6])$ which lies at the heart of his study of the symplectic geometry of the moduli spaces.

Let $X$ be a compact Riemann surface of genus $g>1$, equipped with its Poincaré metric of constant curvature -1 . Let $\Delta_{X}$ be the attending Laplace operator on $X$ and $\left\{\lambda_{n}\right\}_{n \geq 0}$ be its spectrum, that is the sequence of the (positive) eigenvalues of $-\Delta_{X}$ labelled in increasing order and with multiplicities. For any $n \in \mathbf{Z}_{+}$we define as usual the auxiliary spectral numbers $\rho_{n}$ via the equalities $\lambda_{n}=\frac{1}{4}+\rho_{n}^{2}$; true this defines $\rho_{n} \in \mathbf{C}$ only up to sign but we will consider only even functions of the $\rho_{n}$ 's. We let $\mathcal{M}_{g}$ denote the moduli space of Riemann surfaces of genus $g$, viewed as a complex orbifold (see below for more detail).

A lot of work has been devoted to analysing the variation of the spectra of Riemann surfaces. However the information which has been collected to-date mostly concerns the behavior of the low lying eigenvalues as the surface degenerates. More precisely $\mathcal{M}_{g}$ can be compactified into $\overline{\mathcal{M}}_{g}$ where the divisor at infinity $\mathcal{D}=\overline{\mathcal{M}}_{g} \backslash \mathcal{M}_{g}$ classifies the Riemann surfaces with nodes - or stable curves as they are called by algebraic geometers. For any point in $\mathcal{D}$, the corresponding surface can be thought of as a graph with surfaces of lower genera sitting at the vertices, 
such that the total arithmetic genus is $g$. Let now $X_{\varepsilon}$ be a family of surfaces such that as $\varepsilon$ tends to $0, X_{\varepsilon}$ tends to a surface represented by a graph with $k$ vertices $(2 \leq k \leq 2 g-2)$; then as $\varepsilon$ tends to 0 , the first $k$ eigenvalues (counting 0$)$ of $X_{\varepsilon}$ tend to 0 and the $(k+1)$-st one is uniformly bounded away from 0 . In this prototypical situation tools have been developed which make it possible to prove the assertions summarized above and also to study more accurately the behavior of the $k-1$ nonzero but vanishing eigenvalues. We refer to ([1]) for a short history of the development of this line of thought, references, as well as a recent and precise result of this type.

Very little seems to be known about the variation of arbitrary (in particular non vanishing) eigenvalues of arbitrary (in particular non degenerating) surfaces. We refer to ([8]) for results in the case of degenerating surfaces but non vanishing eigenvalues. The formula derived in this note can be used, like the original trace formula, in the vicinity of any surface, not necessarily near the boundary of $\mathcal{M}_{g}$. However, again like the trace formula, it gives global, not individual information on the (variation of the) spectrum. It may be that using well-adapted test functions, in particular functions which are peaked around a given point, one can extract more local information. We postpone to the concluding section some more speculative remarks about possible applications and amplifications and presently recall some additional background material.

Considering again a compact Riemann surface $X$ of genus $g>1$, it can be uniformized as $X=\mathcal{H} / \Gamma$, where $\mathcal{H}$ denotes the Poincaré upper-half plane and $\Gamma \subset P S L(2, \mathbf{R})$ is a hyperbolic Fuchsian group of the first kind. Let $\gamma$ be a closed geodesic on $X$ of length $\ell(\gamma)$; one can also regard $\gamma$ as an element of $\Gamma$ up to conjugacy and the length is given in matrix term via the formula: $\operatorname{trace}(\gamma)=2 \cosh (\ell(\gamma) / 2)$. Lastly we denote by $\mathcal{P}$ the set of oriented primitive closed geodesics, i.e. the set of non divisible conjugacy classes in $\Gamma$. For any $\gamma \in \Gamma$, there are unique $p \in \mathcal{P}$ and $m \in \mathbf{Z}^{*}$ such that $\gamma=p^{m}$; note that we do not distinguish conjugacy classes and representatives in the notation, when no confusion is liable to arise from this abuse.

Selberg's trace formula deals with even "test" functions $h \equiv h(\rho)$, with Fourier transform $\hat{h}(\tau)$ defined by:

$$
\hat{h}(\tau)=\frac{1}{2 \pi} \int_{-\infty}^{+\infty} h(\rho) \cos (\tau \rho) d \rho .
$$

The even test function $h$ has to satisfy certain regularity and growth conditions which we state in the following

\section{Assumptions}

(i) $h$ can be continued into an analytic function over a strip $|\operatorname{Im}(\rho)|<c$ for some $c>\frac{1}{2}$ and it satisfies the estimates $h(\rho)=O\left(|\rho|^{-\beta}\right)$ as well as $h^{\prime}(\rho)=O\left(|\rho|^{-\beta-1}\right)$ for some $\beta>4$, as $\rho$ goes to infinity inside the strip; 
(ii) The Fourier transform $\hat{h}$ satisfies $\hat{h}(\tau)=O\left(e^{-c|\tau|}\right)$ as $\tau$ goes to infinity on $\mathbf{R}$.

Under the above assumptions $h$ satisfies the Selberg trace formula (cf. e.g. [3], section 2.6), to wit:

$$
\begin{aligned}
\sum_{n \geq 0} h\left(\rho_{n}\right)= & (2 g-2) \int_{-\infty}^{+\infty} \rho \tanh (\pi \rho) h(\rho) d \rho \\
& +\sum_{\gamma \in \mathcal{P}} \sum_{m=1}^{\infty} \frac{\ell(\gamma)}{2 \sinh (m \ell(\gamma) / 2)} \hat{h}(m \ell(\gamma))
\end{aligned}
$$

The trace formula (1) depends on the surface $X$, and the aim of this note is so to speak to "take the derivative of (1) w.r.t. $X$ ". To this end we introduce the Teichmüller space $\mathcal{T}_{g}$ associated with (compact) surfaces of genus $g$; the moduli space is then given as the quotient $\mathcal{M}_{g}=\mathcal{T}_{g} / \Gamma_{g}$, where $\Gamma_{g}$ denotes the mapping class group (or Teichmüller modular group) of genus $g$, that is the orbifold fundamental group of $\mathcal{M}_{g}$. We recall but a few definitions in order to fix notation and refer the reader to e.g. [2], [4] or [5] for more information on these objects. So let $S$ denote "the" differentiable, closed, orientable and compact surface of genus $g$. Given a Riemann surface $X$ of genus $g$, a marking of $X$ is given by a diffeomorphism $\phi: S \rightarrow X$, considered up to isotopy. Two marked Riemann surfaces $\left(X_{1}, \phi_{1}\right)$ and $\left(X_{2}, \phi_{2}\right)$ are equivalent if there exists a conformal diffeomorphism $h: X_{1} \rightarrow X_{2}$ such that $\phi_{2}=h \circ \phi_{1}$, again up to isotopy. A point of $\mathcal{T}_{g}$ is an equivalence class $\mathbf{t}=[(X, \phi)]$ of marked Riemann surfaces. We denote by $\pi: \mathcal{T}_{g} \rightarrow \mathcal{M}_{g}$ the canonical projection which consists in forgetting the marking: $\pi(\mathbf{t})=X$.

Any isotopy class $\alpha$ of closed curves on $S$ determines a function $\ell_{\alpha}$ on $\mathcal{T}_{g}$, such that $\ell_{\alpha}(\mathbf{t})=\ell_{X}(\phi(\alpha))$ is by definition the length of the unique geodesic on $X$ which is isotopic to $\phi(\alpha)$. If moreover $\alpha$ is simple, meaning that there exists a simple representative of $\alpha$ (a regular curve in the class of $\alpha$ without self-intersection), it also determines a vector field $\tau_{\alpha}$ on $\mathcal{T}_{g}$, namely the infinitesimal Fenchel-Nielsen twist along $\alpha$ (see [4], [5] or [7]). For any $\mathbf{t} \in \mathcal{T}_{g}$, one can explicitly find $6(g-1)$ simple closed curves $\alpha_{i}, 1 \leq i \leq 6(g-1)$, such that the corresponding $\tau_{\alpha_{i}}(\mathbf{t})$ span the tangent space of $\mathcal{T}_{g}$ at $\mathbf{t}$ (see [7], Corollary 3.5). Recall also that $\mathcal{M}_{g}$ is smooth at $X=\pi(\mathbf{t})$ if $X$ has no nontrivial automorphism, in which case the images $\pi_{*}\left(\tau_{\alpha_{i}}\right)$ span the tangent space of $\mathcal{M}_{g}$ at $X$. Lastly we mention that the differential of $\ell_{\alpha}$ is conjugate to $\tau_{\alpha}$ for the symplectic structure defined by the Weil-Petersson two-form; in other words $\ell_{\alpha}$ is nothing else but Hamilton's function for the vector field $\tau_{\alpha}$ (see section 8.3 of [4] or [7]).

From now on we do not notationally distinguish $\alpha$ from the geodesic which is isotopic to $\phi(\alpha)$ on some marked Riemann surface. An important ingredient in what follows is

Lemma 1 (S. Wolpert, [6]). The variation of the geodesic length along a twist is 
given by:

$$
\tau_{\alpha}(\ell(\gamma))=C(\alpha, \gamma), \quad \text { where } \quad C(\alpha, \gamma)=\sum_{p \in \alpha \cap \gamma} \cos \widehat{(\alpha, \gamma)}
$$

Here $\widehat{(\alpha, \gamma)}_{p}$ denotes the angle of the two intersecting geodesics at $p$, with the following sign convention (see [6]): lift $p$ to $\tilde{p} \in \mathcal{H}$ and assume w.l.o.g. that $\alpha$ lifts to the vertical axis; then $\widehat{(\alpha, \gamma)}_{p}$ is defined to be the angle between this axis (pointing upward) and the half-line tangent to $\gamma$ pointing towards the right halfplane. This convention makes the expression $\cos \widehat{(\alpha, \gamma)}_{p}$ skew symmetric in $(\alpha, \gamma)$, thus also the sum $C(\alpha, \gamma)$.

\section{The infinitesimal trace formula}

The main result of this note is embodied in the following infinitesimal trace formula:

Theorem 2. Let $h$ be an even function satisfying assumptions (i) and (ii) of section 1, and let $\alpha$ be a simple loop on $S$. Then the following identity holds:

$$
\sum_{n \geq 0} \frac{h^{\prime}\left(\rho_{n}\right)}{2 \rho_{n}} \tau_{\alpha}^{ \pm}\left(\lambda_{n}\right)=\sum_{\gamma \in \mathcal{P}} C(\alpha, \gamma)\left(\sum_{m=1}^{\infty} \phi_{h}^{\prime}(m \ell(\gamma))\right)
$$

where $\phi_{h}$ denotes the map $x \mapsto x / 2(\sinh (x / 2))^{-1} \hat{h}(x)$ and $\tau_{\alpha}^{+}$(resp. $\left.\tau_{\alpha}^{-}\right)$denotes the right (resp. left) derivative along the vector field $\tau_{\alpha}$.

The pieces of notation $C(\alpha, \gamma)$ and $\phi_{h}$ have been introduced for convenience only. Explicitly we have:

$$
\phi_{h}^{\prime}(x)=\frac{x / 2}{\sinh (x / 2)} \hat{h}^{\prime}(x)+\frac{1-x / 2 \operatorname{coth}(x / 2)}{2 \sinh (x / 2)} \hat{h}(x) .
$$

Note furthermore that the l.h.s. of (3) can also be written in the form $\sum_{j \geq 0} h^{\prime}\left(\rho_{j}\right) \tau_{\alpha}\left(\rho_{j}\right)$, which is more natural, provided this expression is correctly interpreted; see the remark at the end of section 3 below.

Formula (3), which can be considered as "the derivative of (1) along $\alpha$ ", can be formally deduced from (1) by means of (2). The next two sections are devoted to justifying this derivation by showing the absolute convergence of the l.h.s. (the spectral side) and then of the r.h.s. (the geometric side) of (3). 


\section{The spectral side}

Let $\mathbf{t}_{0}$ be a point of $\mathcal{T}_{g}$ and $\alpha$ a simple closed loop on $S$. We denote by $\mathbf{t}_{\theta} \in \mathcal{T}_{g}$ the point which is obtained from $\mathbf{t}_{0}$ after twisting along $\alpha$ by the angle $\theta$ (see e.g. [4], section 3.2 and pp. 219-221). Let $X_{\theta}=\pi\left(\mathbf{t}_{\theta}\right) ; g_{\theta}$ denotes the Poincaré metric on $X_{\theta}$ and $\Delta_{\theta}$ the Laplace operator on $X_{\theta}$ for the metric $g_{\theta}$. The increasing sequence of the eigenvalues of $-\Delta_{\theta}$ counted with multiplicities reads $0=\lambda_{0}(\theta) \leq \lambda_{1}(\theta) \leq$ $\ldots \leq \lambda_{n}(\theta) \leq \ldots$

Now for any even function $h$ satisfying the assumptions of section 1 , there is a unique function $H_{h}$ which is analytic in a neighborhood of the real half-line ] $-\frac{1}{2},+\infty$ [ and satisfies $H_{h}\left(z^{2}\right)=h(z)$ for $z \in \mathbf{C}$ such that $\left.z^{2} \in\right]-\frac{1}{2},+\infty[$. In this section we show that under the assumptions (i)-(ii) of section 1, the map $\theta \mapsto \sum_{n>0} H_{h}\left(\lambda_{n}(\theta)-\frac{1}{4}\right)$ has left and right derivatives at $\theta=0$ and that the following holds:

$$
\tau_{\alpha}^{ \pm}\left(\sum_{n \geq 0} H_{h}\left(\lambda_{n}(\theta)-\frac{1}{4}\right)\right)=\sum_{n \geq 0} H_{h}^{\prime}\left(\lambda_{n}(\theta)-\frac{1}{4}\right) \tau_{\alpha}^{ \pm}\left(\lambda_{n}\right) .
$$

We first prove:

Lemma 3. The map $\theta \mapsto\left(I-\Delta_{\theta}\right)^{-1}$ is analytic over $\mathbf{R}$, with values in the space of bounded operators on $L^{2}$, endowed with the topology given by the operator norm.

Proof. The last two formulas on p. 133 of [6] explicitly describe a one-parameter family of Beltrami coefficients which define the points $\mathbf{t}_{\theta} \in \mathcal{T}_{g}$. The third formula on p. 134 of that paper gives explicit formula for the corresponding hyperbolic line element $d s$. This formula shows in particular that the dependence of the metric tensor $d s^{2}$ upon $\theta$ is real analytic. Using the expression of the Laplace operator in terms of the metric, one finds that $\left(I-\Delta_{\theta}\right)\left(I-\Delta_{0}\right)^{-1}$ is a linear combination of the four operators $\left(I-\Delta_{0}\right)^{-1}, \partial_{z}\left(I-\Delta_{0}\right)^{-1}, \partial_{\bar{z}}\left(I-\Delta_{0}\right)^{-1}$ and $\partial_{z} \partial_{\bar{z}}\left(I-\Delta_{0}\right)^{-1}$ with coefficients that are real-analytic functions of $\theta$. Since the four operators above are bounded on $L^{2}(S)$, this shows that the map $\theta \mapsto\left(I-\Delta_{\theta}\right)\left(I-\Delta_{0}\right)^{-1}$ is real analytic over $\mathbf{R}$ with values in the algebra of bounded operators on $L^{2}(S)$, equipped with the operator norm. Furthermore, for each $\theta \in \mathbf{R}$ the bounded operator $\left(I-\Delta_{\theta}\right)\left(I-\Delta_{0}\right)^{-1}$ is an invertible element of that algebra. Thus the map $\theta \mapsto\left[\left(I-\Delta_{\theta}\right)\left(I-\Delta_{0}\right)^{-1}\right]^{-1}$ is also real analytic on $\mathbf{R}$, which finishes the proof of the lemma.

As the operator $\Delta_{\theta}$ is self-adjoint on $L^{2}\left(X_{\theta}\right)$ for any $\theta \in \mathbf{R}$, lemma 3 combined with the Kato-Rellich theorem ( $c f$. [9], section XII.13) implies that for any $n \in \mathbf{Z}_{+}$, $\lambda_{n}$ is Lipschitz continuous on $\mathbf{R}$ w.r.t. $\theta$, with left and right derivatives at every point. Writing $\left(\lambda_{n}\right)_{l}^{\prime}(\theta)$ (resp. $\left.\left(\lambda_{n}\right)_{r}^{\prime}(\theta)\right)$ for the left (resp. right) derivative, we have the following estimate: 
Lemma 4. For any $n \in \mathbf{Z}_{+}$and any $\theta \in \mathbf{R}$,

$$
\max \frac{\left[\left|\left(\lambda_{n}\right)_{l}^{\prime}(\theta)\right|,\left|\left(\lambda_{n}\right)_{r}^{\prime}(\theta)\right|\right]}{\left[1+\lambda_{n}(\theta)\right]^{2}} \leq\left\|\frac{d}{d \theta}\left(I-\Delta_{\theta}\right)^{-1}\right\| .
$$

Proof. Let $A_{\theta}=\left(I-\Delta_{\theta}\right)^{-1}$ and $\mu_{n}=1 /\left(1+\lambda_{n}\right)$. Pick $n \in \mathbf{Z}_{+}$and $\theta>0$. Then either $\mu_{n}(\theta)$ belongs to the spectrum $\operatorname{spec}\left(A_{0}\right)$ of $A_{0}$, or it does not. In the former case the operator $I-\left(\mu_{n}(\theta)-A_{0}\right)^{-1}\left(A_{\theta}-A_{0}\right)$ is not invertible, which shows that

$$
1 \leq\left\|\left(\mu_{n}(\theta)-A_{0}\right)^{-1}\right\|\left\|A_{\theta}-A_{0}\right\|=\operatorname{dist}\left(\mu_{n}(\theta), \operatorname{spec}\left(A_{0}\right)\right)^{-1}\left\|A_{\theta}-A_{0}\right\| .
$$

Since $\lambda_{n}$ is known to have a right derivative at $\theta=0$, the above estimate implies that the right derivative $\left|\left(\mu_{n}\right)_{r}^{\prime}(0)\right|$ is bounded from above by the r.h.s. of $(5)$. The proof for the left derivative at 0 and for the other values of $\theta$ is identical.

We now come to the proof of identity (4). For any $n \geq 0$ the function $\theta \mapsto$ $H_{h}\left(\lambda_{n}(\theta)-\frac{1}{4}\right)$ has left and right derivatives at every point $\theta \in \mathbf{R}$. Thanks to assumption (i) the function $H_{h}$ satisfies $H_{h}^{\prime}(x)=h^{\prime}(\sqrt{x}) / 2 \sqrt{x}=O\left(x^{-\beta / 2-1}\right)$ for some $\beta>4$, so that using the estimate (5) we find that

$$
\sup \left[\left|\left(\lambda_{n}\right)_{g}^{\prime}(\theta)\right|,\left|\left(\lambda_{n}\right)_{d}^{\prime}(\theta)\right|\right]\left|H_{h}^{\prime}\left(\lambda_{n}(\theta)-\frac{1}{4}\right)\right|=O\left(\lambda_{n}(\theta)^{1-\beta / 2}\right) .
$$

Now the Weyl asymptotic estimate $\lambda_{n}(\theta)=O(n)$ is locally uniform w.r.t. $\theta$; the estimate above, combined with this fact and the dominated convergence theorem vindicates formula (4).

Remark. By the Kato-Rellich theorem quoted above, there exists in fact a relabeling $\sigma: \mathbf{Z}_{+} \rightarrow \mathbf{Z}_{+}$of the spectrum such that for any $j \in \mathbf{Z}_{+}$the map $\theta \mapsto \lambda_{\sigma(j)}(\theta)$ is real analytic in $\theta$ over some $j$-dependent neighborhood of $\theta=0$. So for $\theta=0$ we may in fact write:

$$
\sum_{n \geq 0} H_{h}^{\prime}\left(\lambda_{n}-\frac{1}{4}\right) \tau_{\alpha}^{ \pm}\left(\lambda_{n}\right)=\sum_{j \geq 0} h^{\prime}\left(\rho_{\sigma(j)}\right) \tau_{\alpha}\left(\rho_{\sigma(j)}\right),
$$

in which $h^{\prime}\left(\rho_{\sigma(j)}\right) \tau_{\alpha}\left(\rho_{\sigma(j)}\right)$ is interpreted as $\frac{h^{\prime}\left(\rho_{\sigma(j)}\right)}{\rho_{\sigma(j)}} \tau_{\alpha}\left(\rho_{\sigma(j)}^{2}\right)$. Indeed since $h$ is even and analytic in a neighborhood of the real axis, this is also the case for the function $h^{\prime}(z) / z$.

\section{The geometric side}

We turn to the r.h.s. of the variational trace formula (3), with the aim of proving the identity:

$$
\tau_{\alpha}\left(\sum_{\gamma \in \mathcal{P}} \sum_{m=1}^{\infty} \phi_{h}(\gamma)\right)=\sum_{\gamma \in \mathcal{P}} C(\alpha, \gamma)\left(\sum_{m=1}^{\infty} \phi_{h}^{\prime}(m \ell(\gamma))\right)
$$


Given (2) (see Wolpert's lemma in section 1) this amounts to justifying the switching of the sum and the derivative $\tau_{\alpha}$. To this end we need to bound the intersection term $C(\alpha, \gamma)$ from above. Given the closed geodesics $\alpha$ and $\gamma$, let $I(\alpha, \gamma)$ be the number of their intersection points; then clearly $|C(\alpha, \gamma)| \leq I(\alpha, \gamma)$, so that it suffices to bound the last quantity, with the additional assumption that $\alpha$ is simple. In order to do this we need some elementary hyperbolic geometry and first define the quantity $\eta_{m}=\eta_{m}(X, \alpha)>0$ as follows. Given $\eta \geq 0$, let $B_{\alpha}(\eta)$ be the open strip of width $2 \eta$ centered around $\alpha$, in other words the set of points of $X$ whose distance to $\alpha$ is strictly smaller than $\eta$. We define $\eta_{m}$ as the supremum of those $\eta>0$ such that $B_{\alpha}(\eta)$ is isometric to the standard annulus of width $2 \eta$. To put it slightly differently, for any $\eta<\eta_{m}$ and any point $x \in B_{\alpha}(\eta)$, there is a unique geodesic segment contained in $B_{\alpha}(\eta)$, originating at $x$ and perpendicular to $\alpha$; moreover $\eta_{m}$ is maximal with this property.

Then we have the following estimate:

Lemma 5. $I(\alpha, \gamma) \leq \ell(\gamma) /\left(2 \eta_{m}\right)$

Proof. Let $p \in \alpha \cap \gamma$ and $\eta<\eta_{m}$; then $\gamma$ traverses the strip $B_{\alpha}(\eta)$ and defines there a geodesic segment which we denote by $I_{p}$. Clearly the length of $I_{p}$ is at least $2 \eta$. Moreover as $p$ runs along the finite set $\alpha \cap \gamma$, the segments $I_{p}$ are disjoints, except possibly for a finite number of transverse intersections corresponding to self-intersections of $\gamma$ located in the strip $B_{\alpha}(\eta)$. The lemma follows from these considerations.

Remarks. There is a universal lower bound for $\eta_{m}$, depending only on the length of the simple geodesic loop $\alpha$. In fact one can show that $\eta_{m}(\alpha, X) \geq \eta_{0}(\ell(\alpha))$, where the function $\eta_{0}$ is defined by the equality $\cosh \left(\eta_{0}(\ell)\right)=1 / \tanh (\ell / 2)$ ( $\operatorname{see}$ [5], section I.1.5).

One can also notice that because two geodesics always intersect transversally, the intersection number $I(\alpha, \gamma)$ is in fact constant over $\mathcal{T}_{g}$. Moreover this constant coincides with the topological intersection number of the isotopy classes $\alpha$ and $\gamma$ on the model topological surface $S$. Recall that this number is defined as the minimal number of intersection points of two representatives of the classes $\alpha$ and $\gamma$. The fact that $I(\alpha, \gamma)$, which was first defined via hyperbolic geometry, coincides with that purely topological quantity is one of the basic results in Thurston's theory of surfaces, leading in particular to his compactification of Teichmüller spaces (see [10], Exposé 3).

We come to the by now easy proof of (6). Wolpert's lemma recalled in section 1 ensures that $\left|\tau_{\alpha}(l(\gamma))\right|=|C(\alpha, \gamma)| \leq I(\alpha, \gamma)$, and this last quantity is bounded from above as in lemma 5. On the other hand, assumptions (i)-(ii) on the test function $h$ show that $\phi^{\prime}(m l(\gamma))=O\left(e^{-c^{\prime} m l(\gamma)}\right)$ for all $\left.c^{\prime} \in\right] 1, c+\frac{1}{2}[$. The dominated convergence theorem then justifies term-by-term differentiation of the r.h.s. of (6), 
finishing the proof of the validity of (6).

Putting together the results of section 3 and section 4 finishes the proof of the theorem, showing the validity of (3).

\section{Amplifications, possible applications, remarks}

Let us close with some of the remarks the above might trigger. First we note that we have adopted the point of view of hyperbolic geometry along with the real analytic description of the Teichmüller and moduli spaces. One can at least partially introduce the conformal viewpoint for surfaces along with the complex analytic theory for the classifying spaces by replacing (2) with an analogue in terms of Poincaré series (see in particular [7], theorem 2.9).

The input on the geometric side of the trace formula (1) is the length spectrum of the surface, that is the collection of the numbers $\ell(\gamma)$ for all primitive geodesics $\gamma \in \mathcal{P}$ (counting with multiplicities). By a classical remark originally due to H. Huber and A. Selberg, the trace formula itself implies that the spectrum and the length spectrum determine each other. Now working around a given surface $X$, the input of the variational trace formula (3) consists of either of the two spectra supplemented by the geometric information conveyed by the numbers $C(\alpha, \gamma)$. Now for any test function $h$ satisfying the assumptions stated in section 1, (3) provides a linear relation between the derivatives $\tau_{\alpha}^{ \pm}\left(\lambda_{n}\right)$ (see also the remark at the end of section 3 ). Varying $h$, one finds that (3) actually determines the collection of these numbers, i.e. it determines so to speak the derivative of the spectrum of the surface along any given twist. So either of the length spectrum or the spectrum, supplemented with the intersection data $C(\alpha, \gamma)$ for all pairs of primitive geodesics $\alpha, \gamma \in \mathcal{P}$ determine both the derivatives of the length spectrum (by lemma 1) and of the spectrum (by (3)).

We have discussed only the comparatively elementary case of closed Riemann surfaces. From a more general viewpoint the above is certainly a rank 1 phenomenon, because in higher ranks rigidity results precisely say that the analog simply does not exist. It would however be interesting to investigate the possibility of adapting the above to other base fields (still rank 1 but not over the complex numbers). Coming back to Riemann surfaces (alias complex curves) one can add elliptic elements for free, because these are rigid. In other words the first term on the r.h.s. of (1) corresponds to the identity element of the uniformizing group $\Gamma$ and disappears upon taking the derivative, as it is independent of the curve. In exactly the same way the additional terms which appear when $\Gamma$ is not assumed to be torsion free depend only on the signature of the group (see e.g. [11], theorem 9.1 with $\chi=1$ ); so they also disappear in the variation. The extension to the noncompact case however is of course highly non trivial and hopefully interesting, and so is the degenerating process corresponding to - depending on the viewpointsqueezing a simple closed geodesic, going to the boundary of the moduli space and 
the emergence of parabolic elements in the uniformizing Fuchsian group.

It is to be hoped that (3) and possible extensions or variations therof can be useful in several situations. Here are a few tracks, including possible dead ends. One may want to study the variation of the Selberg zeta function or other generating functions. The formula can in principle be iterated or even exponentiated, so as to get information about what happens along a finite twist (see [7], theorem 3.4). One can also try to investigate various bifurcation phenomena such as the study of the spectrum near a surface with nontrivial automorphisms, or the splitting of a multiple eigenvalue as the surface varies.

Acknowledgements. We thank Eric Leichtnam and the referee for helpful remarks.

\section{References}

[1] P. Batchelor, Dérivée des petites valeurs propres des surfaces de Riemann, Comment. Math. Helvetici 73 (1998), 337-352.

[2] P. Buser, Geometry and Spectra of Compact Riemann Surfaces, Birkhäuser Verlag, 1992.

[3] P. Cartier and A. Voros, Une nouvelle interprétation de la formule des traces de Selberg, in: P. Cartier et al. eds., The Grothendieck Festschrift, Volume II, 1-66, Progress in Mathematics 87, Birkhäuser, 1990.

[4] Y. Imayoshi and M. Taniguchi, An introduction to Teichmüller spaces, Springer Verlag, 1992.

[5] X. Buff, J. Fehrenbach and P. Lochak, Éléments de géométrie des espaces de modules de courbes, in: Espaces de modules de courbes, groupes modulaires et théorie des champs, Panoramas et Synthèses 7, Publications SMF, 1999.

[6] S. Wolpert, An elementary formula for the Fenchel-Nielsen twist, Comment. Math. Helvetici 56 (1981), 132-135.

[7] S. Wolpert, The Fenchel-Nielsen deformation, Annals of Math. 115 (1982), 501-528.

[8] S. Wolpert, Spectral limits for hyperbolic surfaces, I and II, Invent Math. 108 (1992), 67-89 and $90-129$.

[9] M. Reed and B. Simon, Methods of Modern Mathematical Physics, Vol. 4, Academic Press, 1978.

[10] Travaux de Thurston sur les surfaces, Astérisque 66-67, A. Fathi, F. Laudenbach and V. Poenaru eds., SMF Publ., 1979 (2nd edition 1991).

[11] A. B. Venkov, Spectral theory of automorphic functions, Russian Math. Surveys 34 (1979), $79-153$.

F. Golse

Institut Universitaire de France

\& Laboratoire Jacques-Louis Lions

175 rue du Chevaleret

75013 Paris

France

e-mail: golse@math.jussieu.fr

(Received: May 7, 2001)
P. Lochak

Institut Mathématique de Jussieu

175 rue du Chevaleret

75013 Paris

France

e-mail: lochak@math.jussieu.fr 